\title{
La réaction à l'énoncé ironique dans un corpus théâtral
}

\author{
Elodie Baklouti \\ Université Montpellier 3, Praxiling (UMR 5267) \\ Elodie.baklouti@univ-montpellier3.fr
}

Résumé. Au sein des très nombreux travaux portant sur l'ironie verbale recensés jusqu'ici, les études traitant de la réaction à l'énoncé ironique sont rares, si ce n'est, inédites, en raison peut-être de la difficulté de l'accès à un corpus offrant la possibilité de décrire cette dimension réceptive. Le texte théâtral, genre de la mimesis conversationnelle, en raison de sa structure dialogale nous semble permettre d'appréhender l'énoncé ironique dans son amont et dans son aval, en tant que phénomène interactionnel. Il est possible d'y examiner l'intervention réactive à l'acte ironique sur les plans sémantico-pragmatiques. Dans notre conception de l'énoncé ironique en tant qu'acte indirect d'attaque, nous entendons analyser les différents modes d'enchaînements que peut mettre en œuvre le locuteur dans un corpus constitué d'extraits de pièces de théâtre. Nous examinerons la façon dont le tour faisant suite à l'énoncé ironique nous renseigne sur l'interprétation de celui-ci par l'interlocuteur et tenterons également de déterminer les divers ressorts que peut mobiliser le sujet parlant pour gérer l'attaque dont il fait l'objet lorsque celle-ci est non inscrite implicitement dans l'énoncé, cette non-inscription explicite de la critique pouvant, le cas échéant, se retourner contre celui qui l'a formulée.

\begin{abstract}
Among the numerous studies on verbal irony identified so far, the studies dealing with the reaction to the ironic statement are scarse, due perhaps to the difficulty of access to a corpus in which it's possible to describe the receptive dimension. The theatrical text, kind of conversational mimesis, owing to its dialogical structure, may allow us to understand the ironic statement in its upstream and its downstream, as interactional phenomenon. It is possible to examine the reactive answer to the ironic act on semanticpragmatic plans. In our conception of ironic statement as an indirect attack act, we intend to analyze the different modes of reactions that the speaker can implement, in a corpus made up of extracts of plays. We will examine how the turn following the ironic statement provides information on the interpretation thereof by the interlocutor and also try to determine the various means that the speaker can mobilize to handle the attack of which he is the object when this attack is not implicitly included in the statement, the explicit noninclusion of criticism can, if necessary, turn against one who has made it.
\end{abstract}

\section{Introduction}

Au sein des très nombreux travaux portant sur l'ironie verbale recensés jusqu'ici, les études traitant de la réaction à l'énoncé ironique sont rares, si ce n'est, inédites, en raison peut-être de la difficulté de l'accès à un corpus offrant la possibilité de décrire cette dimension réceptive. Le texte théâtral, genre de la mimesis conversationnelle, en raison de sa structure dialogale nous semble permettre d'appréhender l'énoncé 
ironique dans son amont et dans son aval, en tant que phénomène interactionnel. Il est possible d'y examiner l'intervention réactive à l'acte ironique sur les plans sémantico-pragmatiques. Dans notre conception de l'énoncé ironique en tant qu'acte indirect d'attaque, nous entendons analyser les différents modes d'enchaînements que peut mettre en œuvre le locuteur dans un corpus constitué d'extraits de pièces de théâtre. Nous examinerons la façon dont le tour faisant suite à l'énoncé ironique nous renseigne sur l'interprétation de celui-ci par l'interlocuteur et tenterons également de déterminer les divers ressorts que peut mobiliser le sujet parlant pour gérer l'attaque dont il fait l'objet lorsque celle-ci est non inscrite implicitement dans l'énoncé, cette non-inscription explicite de la critique pouvant, le cas échéant, se retourner contre celui qui l'a formulée.

Notons que les données exploitées ne sont pas spontanées et authentiques. Mais il nous semble qu'une étude du texte théâtral peut venir alimenter les réflexions générales sur l'objet saisissant qu'est l'ironie. Rappelons à ce titre les propos de Barthes et Berthet (1979:4) :

\begin{abstract}
Chaque fois, donc, que les sciences sociales ont à traiter d'un objet de langage (ou, pour être plus précis, d'un "discours"), elles auraient bien tort de ne pas recourir au corpus littéraire; sans doute, sauf exception (nous pensons à Proust), elles n’y trouveront pas des "analyses", des "explications", mais, en contrepartie, des descriptions, des reproductions, des simulacres, si bien agencés, que l'intelligence première du propos se double virtuellement d'une intelligence théorique et comme structurale du langage lui-même.
\end{abstract}

Nous avons délimité notre corpus à partir de la base de données Frantext grâce à laquelle nous avons pu sélectionner des extraits d'œuvres théâtrales dans lesquels apparaissaient les termes «ironie, ironique, ironiser, ironiquement », sous forme de didascalie pour caractériser métadiscursivement une réplique. Par ce type de constitution du corpus dans lequel le linguiste n'est pas à l'origine de la catégorisation en tant qu'énoncé ironique, nous tentons de nous départir d'une démarche fondée sur des critères subjectifs.

\title{
2 Point théorique sur l'ironie
}

Nous le disions supra, il existe de nombreux travaux prenant pour objet l'ironie. Au sein de ceux-ci, on peut distinguer globalement quatre grands types d'approche.

La plus ancienne, dite conception classique, issue de la tradition rhétorique (Quintilien (95), Dumarsais (1730), Fontanier (1977), Kerbrat-Orecchioni (1980) etc.), considère l'ironie comme une antiphrase : elle consisterait à faire entendre le contraire de ce qu'elle dit.

Cette conception a été souvent jugée insuffisante voire superficielle par certains linguistes ( Martin, 1983) dont Sperber et Wilson qui proposent une approche innovante en 1978 dite théorie échoïque en ce sens que l'écho y occupe une fonction fondamentale (1978:409) : dans l'ironie «le locuteur fait écho à une proposition d'une manière propre à manifester qu'il la désapprouve soit parce qu'elle manque de vérité (et partant de pertinence), soit parce qu'elle manque directement de pertinence ».

Un autre courant porté par Clark et Gerrig (1984) puis Currie (2006) estime que la spécificité de l'ironie réside dans la pretence ou feintise en français : l'ironiste ferait semblant de tenir un énoncé qu'il ne prend pas véritablement en charge.

Enfin, on peut considérer un grand ensemble d'approches pragmatico-énonciatives (Ducrot 1984, Perrin 1996, etc.), que l'on nommera approches mixtes selon lesquelles l'ironie constitue un phénomène composite couplant à d'autres paramètres ceux de l'écho et de la feintise, phénomène qui trouve selon la conception concernée diverses appellations : double-jeu énonciatif (Rabatel, 2012), cocktail dialogique (Bres, 2010), paradoxe argumentatif (Berrendonner, 1981) etc.

Nous considérons, nous, que l'ironie consiste fondamentalement en un acte indirect d'attaque. Tous les éléments mis en avant par les différentes conceptions évoquées (écho, feintise, antiphrase...) sont autant de procédés permettant de conférer à l'ironie cette indirection. Cet acte indirect (dérivé ou implicite) de 
reproche, d'attaque, de critique est produit sous le couvert d'un autre acte, dit primitif, direct ou encore explicite. Ces précisions théoriques faites, venons-en à l'analyse.

\section{Structuration générale de l'énoncé ironique}

Nous avons pu observer que l'échange dans lequel surgit l'énoncé ironique se présente dans la plupart des cas de la façon suivante :

- énoncé de L1 perçu comme manquant de pertinence par L2 et pouvant comporter une forme d'attaque de L1 envers L2 (L2 est mis en position de dominé par rapport à L1)

- énoncé réactif ironique de L2 qui constitue une attaque-retour envers L1 (L2 inverse le rapport de force)

- réaction de L1 ou d'un autre personnage solidaire de L1 se sentant lui-même attaqué par L2, qui peut enchaîner :

- (1) soit sur la valeur indirecte de reproche/ critique/ d'attaque contenue dans l'énoncé de L2

- (2) soit sur la valeur directe, dénotée contenue dans l'énoncé de L2

- (3) soit en produisant une réponse ambiguë interprétable à la fois comme enchaînement sur la valeur directe et sur la valeur indirecte de l'énoncé de L2

Ce sont précisément à ces trois différents modes d'enchaînement par L1 à l'énoncé ironique de L2 que nous nous intéresserons dans ce travail.

\section{Enchaînement sur la valeur indirecte transmise par l'énoncé ironique}

\subsection{Enchaînement sur la valeur indirecte de l'énoncé ironique non conventionnel}

L'interlocuteur peut enchaîner sur la valeur indirecte contenue dans l'énoncé de L2. Kerbrat-Orecchioni parle dans ce cas de trope illocutoire en ce sens qu'il (1986:76) « opère un renversement de la hiérarchie des niveaux de contenus et se caractérise par un évincement du contenu primitif par le contenu dérivé ».

C'est ce qui se produit ici :

(1) LE PROMOTEUR, déguisé, se penchant vers Ida. - C'est bien la première fois que je me mêle ainsi à la foule.

IDA, ironique et montrant la place déserte. - La foule ! Si l'on peut dire !

LE PROMOTEUR. - Même s'il n'y a personne ici, on pressent qu'il y a beaucoup de monde... ailleurs.

J. TARDIEU, La Cité sans sommeil, 1984

Dans cet exemple, la réponse du promoteur « même s'il n'y a personne ici, on pressent qu'il y a beaucoup de monde... ailleurs » enchaîne sur l'acte dérivé produit par l'énoncé de Ida. En effet, la réplique d'Ida, soit L2, était destinée à pointer le manque de pertinence de l'énoncé du promoteur, soit L1 : une discordance s'instaure entre le mot «foule » et ce à quoi le mot réfère contextuellement à savoir «la place déserte» pointée non pas verbalement mais gestuellement par la monstration d'Ida. Cette inadéquation est également signalée par la boucle métaénonciative " si l'on peut dire » qui relève de la non-coïncidence entre les mots et les choses selon J. Authier-Revuz. Il s'agit d'une boucle posant « un dire suspendu à sa possibilité » (1995: 632) par la subordonnée circonstancielle de condition et l'auxiliaire modal «pouvoir ». La nomination par le substantif foule ne se plie pas aux règles d'association mot-chose. En effet, le rapport qui unit le mot «foule » à la situation (place déserte) procède du paradoxe. S'il est un "consensus d'une norme de fonctionnement souple du rapport motchose» (op. cit : 556) laissant un espace ouvert dans le dire «permis », la nomination choisie par le 
promoteur n'y entre pas. Le mot n'entretient pas de lien, même minimal, avec la situation et trahit une forme de folie ou en tout cas de déni de la réalité par son locuteur. L'énoncé d'Ida était une assertion affirmative elliptique dont on peut reconstituer le contenu explicite «oui il y a foule!». Son sens implicite est délivré en mettant l'assertion à la modalité négative. Sens sous-entendu que la subordonnée circonstancielle concessive «même s'il n'y a personne ici » reprend sous forme présupposé «il n'y a personne ».

L'occurrence (2) correspond au même type de configuration :

(2) DORANTE. - J'ai lieu de présumer que mes services ne vous sont plus agréables, et dans la conjoncture présente, il est naturel que je sache mon sort.

MADAME ARGANTE, ironiquement. - Son sort ! Le sort d'un intendant : que cela est beau !

MONSIEUR REMY. - Et pourquoi n'aurait-il pas un sort ?

ARAMINTE, d'un air vif à sa mère. - Voilà des emportements qui m'appartiennent. (à Dorante.) Quelle est cette conjoncture, Monsieur, et le motif de votre inquiétude ?

MARIVAUX, Les Fausses Confidences, 1738

La réplique de Monsieur Remy « et pourquoi n'aurait-il pas un sort ? » enchaîne sur la valeur dérivée délivrée par l'énoncé de Madame Argante «son sort! Le sort d'un intendant que cela est beau ». Cet énoncé exclamatif fait état d'un constat positif utilisant l'adjectif axiologique mélioratif «beau » se rapportant au syntagme «le sort d'un intendant». Il véhicule un acte dérivé de négation "vous n'avez pas de sort/ un intendant n'a pas de sort/ on ne parle pas de sort pour un intendant/ ». Il s'agit de nier implicitement le présupposé contenu dans la réplique d'Argante. En effet quand ce dernier s'écrie "il est naturel que je sache mon sort », il présuppose qu'il en possède un. Présupposé qui ne va pas du tout de soi pour Madame Argante. C'est bien sur le sens dérivé d'assertion négative transmis par l'acte d'exclamation positive de Madame Argante que Monsieur Rémy enchaîne par sa question partielle «Et pourquoi n'aurait-il pas un sort ? » s'appuyant sur le présupposé « il n'a pas de sort ».

(3) GARCIN. - où est l'interrupteur ?

LE GARÇON. - Il n'y en a pas

GARCIN. - Alors on ne peut pas éteindre?

LE GARÇON. - La direction peut couper le courant mais je ne me rappelle pas qu'elle l'ait fait à cet étage-ci. Nous avons l'électricité à discrétion.

GARCIN. - très bien. Alors il faut vivre les yeux ouverts...

LE GARÇON, ironique. - vivre..

GARCIN. - vous n'allez pas me chicaner pour une question de vocabulaire. Les yeux ouverts. Pour toujours. Il fera grand jour dans mes yeux. Et dans ma tête.

J-P. SARTRE, Huis-clos, 1944

Dans l'exemple (3), la réplique en gras du garçon est destinée à pointer la non-coïncidence entre les propos de Garcin « « il faut vivre les yeux ouverts » dont il reprend en écho le terme vivre et la réalité : les personnages sont morts, Garcin ne peut donc pas utiliser le mot « vivre » pour décrire leur réel, la discordance entre le mot et la situation est même de l'ordre du paradoxe, vie et mort s'inscrivant dans un rapport antinomique.

La réplique du Garçon qui prend la forme de l'énoncé elliptique "vivre » à valeur primitive d'assertion affirmative «il faut vivre/vous avez dit vivre» prend le sens d'une assertion négative à valeur de réfutation et donc de critique « vivre n'est pas le mot/ vous vous trompez en disant vivre/». Contestation que Garcin perçoit puisqu'il catégorise cette réplique du Garçon comme une façon de le « chicaner » et enchaîne sur la valeur dérivée de contestation et donc de critique de l'énoncé. Garcin utilise ainsi le verbe «chicaner» associé au pronom complément de première personne «me » référant à lui-même, en fonction de complément d'objet. Cette construction signifie " créer des difficultés », « quereller au sujet d'un élément jugé insignifiant pour le locuteur » (TLFi) ${ }^{i}$, ici Garcin. L'élément jugé insignifiant étant en fonction de complément circonstanciel de but introduit par la préposition «pour » : "pour une question de vocabulaire ». Cette caractérisation métadiscursive " pour une question de vocabulaire » signale bien que c'est le mot choisi par Garcin qui a été remis en cause par le garçon. 
(Géronte envisage de marier sa fille, Julie. Sa sœur, la comtesse qui a une grande influence sur lui, voudrait qu'il lui choisisse pour époux le marquis. Mais Géronte semble avoir été séduit par Dorante.)

(4) GÉRONTE . - le Marquis... Je vous avoue, ma sœur, que plus j’y pense et moins je puis me déterminer à le préférer à Dorante.

LA COMTESSE, ironiquement. - Dorante ?

GÉRONTE. - Je sais, comme vous, qu'il a des façons de penser très-extraordinaires, et qu'il soutient des thèses...

LA COMTESSE, l'interrompant, plus ironiquement encore. - Dorante, Monsieur ?

GÉRONTE. - Mais il joint un bien considérable à une grande naissance.

LA COMTESSE, en haussant les épaules. - Dorante!

B-J. SAURIN, Les Mours du tems, 1761

En (4), l'interrogation de la comtesse «Dorante ?» acquiert une valeur de désaccord avec l'énoncé précédemment tenu par Géronte qui avoue préférer Dorante au marquis. L'enchaînement de Géronte «je sais comme vous... » par lequel il justifie son choix nous indique qu'il a compris l'interrogation de sa sœur comme une désapprobation. Il concède à sa sœur par sa première proposition « je sais... " que Dorante a des défauts, il se présente donc dans un premier temps en accord avec l'opposition implicite qu'elle vient de produire puis produit une seconde proposition introduite par la conjonction de coordination « mais » qui expose un argument présenté comme supérieur anti-orienté à l'argumentation supportée dans la première proposition. Géronte développe donc son argumentation comme si la comtesse faisait état explicitement de sa divergence d'opinion, c'est bien sur la valeur dérivée des questions qu'il enchaîne.

L'enchaînement sur l'acte dérivé est possible nous l'avons dit si l'interlocuteur perçoit l'énoncé comme implicite. Dans les cas que nous venons de voir, les énoncés interprétés comme « ironiques » n'avaient pas une valeur antiphrastique inscrite en langue. Ainsi l'énoncé «le sort d'un intendant: que cela est beau »n'a pas une valeur axiologique négative immuable quel que soit le contexte. Pour autant, il existe bien des expressions que l'on nomme à la suite de Kerbrat-Orecchioni «conventionnelles » et qui déclenchent communément la lecture en tant qu'acte indirect comme « un beau parleur», " (se/me) faire une belle jambe »...etc. Dans ces cas, l'interlocuteur enchaîne toujours sur la valeur dérivée de l'énoncé. C'est ce qui se produit avec l'exemple suivant.

\subsection{Enchaînement sur la valeur indirecte de l'énoncé ironique conventionnel}

(5) PROMOTEUR. - Même s'il n'y a personne ici, on pressent qu'il y a beaucoup de monde... ailleurs. (Il désigne d'un geste l'horizon.)

LE CHEF DU C.S.G, ironique. -Vous ne perdez rien pour attendre : on va laisser entrer quelques personnes. Vous pourrez les écouter (avec une sombre ironie), constater l'affection des gens pour leur chef bien-aimé ! (Il sort par la gauche.) LE PROMOTEUR, contenant sa fureur et désignant la direction par où vient de disparaître le policier. - Celui-là, quand les choses seront rentrées dans l'ordre, je le fais pendre!

J. TARDIEU, La Cité sans sommeil, 1984

La réplique du chef du CSG contient le tour «vous ne perdez rien pour attendre » que l'on peut considérer comme une expression antiphrastique lexicalisée. Si originellement, elle signifiait que l'attente n'était pas préjudiciable et pouvait même devenir un avantage, elle a univoquement aujourd'hui le sens d'une menace. Le promoteur ne peut donc qu'enchaîner sur cette valeur dérivée de menace : la didascalie décrit sa « fureur » et son énoncé fait état du châtiment funeste qu'il réserve au chef de la CSG. Il a donc bien reçu comme une attaque/menace cette réplique du CSG. Notons cependant que l'ironie de cette réplique n'est pas uniquement contenue dans cette expression. La deuxième didascalie « avec une sombre ironie » redouble la catégorisation et signale que le segment discursif qui la suit est ironique également.

Ainsi il existe certaines expressions lexicalisées qui conditionnent ou du moins orientent fortement l'enchaînement sur la valeur dérivée de l'énoncé ironique. 


\section{Enchaînement sur la valeur directe, dénotée contenue dans l'énoncé de L2}

L'interlocuteur peut également enchaîner sur la valeur directe, dénotée de l'énoncé. Le sens implicite est éludé. Kerbrat-Orecchioni parle pour ces cas de «dérivation allusive » (op. cit :76). L1 peut produire ce type d'enchaînement pour plusieurs raisons. Il peut tout simplement ne pas avoir saisi la critique dissimulée sous l'énoncé.

\subsection{Non compréhension de la valeur d'attaque : l'ironie se fait aux dépens de l'interlocuteur}

(6) CHANAL. - Qu'est-ce qu'elle fait là, celle-là ? c'est pas sa place ! (Il prend la petite table, puis, tout en la portant au fond :) Ah! la, la, la ! la !

ETIENNE, arrivant du fond; il est en veston de travail en coutil mauve. - C'est Monsieur qui a sonné ?

CHANAL. - C'est moi, oui! (Après un petit temps.) Vous êtes bien sûr que Madame doit rentrer?

ETIENNE, au-dessus du piano. - Oh ! sûr, Monsieur... pour déjeuner. D'ailleurs, Madame m'a bien recommandé pour Monsieur ; elle m'a dit : «Monsieur mon ancien mari doit venir vers une heure, vous le ferez attendre. "

CHANAL, avec une intention ironique. - «Vous » le ferez attendre ? ou « je» le ferai attendre?

ETIENNE, sans comprendre l'ironie. - « Vous » le ferez attendre.

G. FEYDEAU, La Main passe, 1904

La didascalie «sans comprendre l'ironie » signale explicitement la non-compréhension par Étienne de l'intention ironique de Chanal. Étienne, le domestique, n'est pas au fait des éléments faisant partie de l'histoire conversationnelle liant Chanal à son ex-femme. Cette dernière l'a trompé et quitté. Chanal introduit un jeu reposant sur les pronoms personnels déictiques «vous » et «je » dont les références pourraient être rendues opaques en ce qu'ils sont mobilisés dans un discours rapporté direct. Chanal fait ainsi semblant de s'assurer qu'Étienne a bien utilisé le pronom adéquat lorsqu'il a formulé ce discours rapporté. Il s'agit en fait pour Chanal de suggérer que son ex-femme fait exprès de le faire attendre. En d'autres termes, le tour factitif transitif «faire attendre » avec pour sujet le pronom personnel «je» renvoyant à l'ex-femme prend le sens de «tarder à venir, reculer une rencontre » (TLFi) qui suggère que l'actant néglige volontairement la ponctualité. En revanche le même tour, lorsqu'il a pour sujet le pronom personnel « vous » renvoyant à Étienne qui est le domestique, prend le sens mondain de « prier d'attendre dans une antichambre » (TLFi). Si le premier sens caractérise une action qui peut être reprochée à la dame et met en avant son indélicatesse, son impudence, le second sens atteste d'un respect des usages et de la bienséance.

Étienne ne peut percevoir que Chanal adresse ainsi par cette fausse demande de précision un reproche à son ex-femme.

(7) MONSIEUR. - C'est - mais vous n'allez pas vous formaliser ?

LE VOLEUR. - Non, non, parlez sans crainte!

MONSIEUR. - C'est la façon dont ma femme vous... utilise !

LE VOLEUR, avec une ironie à peine sensible. -C'est pour mon bien, vous l'avez

dit vous-même !

MONSIEUR, secouant la tête. - Non, un tel accaparement! Ce despotisme !...

Voyons un jeune homme de votre âge ne devrait plus se laisser ainsi conduire par une femme! Vous n'êtes plus un collégien, que diable!

J. TARDIEU, Le Petit voleur rêve de banlieue, 1984

Après la réplique du Voleur «c'est pour mon bien vous l'avez dit vous-même », Monsieur produit un énoncé négatif commençant par l'adverbe de négation «non»: «Non, un tel accaparement! Ce despotisme !... Voyons... ». Les énoncés exclamatifs, la locution interjective « que diable » marquant son émotion vive suggèrent que Monsieur éprouve une certaine révolte devant la conduite de sa femme à 
l'égard du voleur. Cette révolte est aussi celle qu'il ressent face à l'apparent aveuglement du voleur, qui ne se soulève pas contre la tyrannie qu'exerce Madame sur lui. Monsieur par le verbe voir à la première personne du pluriel de l'impératif « voyons » qui fonctionne comme une particule énonciative, appelle le voleur à partager son constat, à « ouvrir les yeux ». Le verbe devoir au conditionnel à la modalité négative énonce ce qui est contraire à l'ordre des choses, à savoir l'asservissement dont est victime le voleur. Monsieur ne réagit ainsi qu'à l'acte primitif d'assertion affirmative que contient la réplique du voleur «c'est pour mon bien... ». Cet énoncé constitue explicitement un jugement positif du comportement de Madame qui n'agirait que par souci du bien-être du voleur. Monsieur ne réalise pas que le voleur ne fait que faire écho aux propos qu'il avait lui-même tenus quelques répliques avant «si elle vous rudoie un peu, c'est pour votre bien ». Monsieur tenait un discours contraire dans lequel il prenait la défense de sa femme et justifiait le comportement de celle-ci envers lui. Cette réplique sonne donc comme une critique indirecte mettant en contradiction les paroles de Monsieur et que l'on peut représenter comme « ce n'est pas pour mon bien et cela ne l'a jamais été contrairement à ce que vous disiez, vous êtes incohérent ». Monsieur semble ne pas avoir perçu cette dimension d'attaque : s'il cherche à susciter chez le voleur un sentiment de rébellion c'est en effet qu'il estime que le voleur n'est pas conscient du fait qu'il est exploité par sa femme. Monsieur exhorte le voleur à réagir. Il cherche à susciter chez lui une prise de conscience, ce qui nous indique qu'il pense que le voleur n'a pas réalisé que sa femme l'utilise. Ainsi Monsieur semble bien avoir pris « au pied de la lettre » ce jugement positif tenu de façon feinte par le voleur.

(8) CLARISSE, lui coupant la parole. - Oh ! je sais, tout t'est dû ! Un remerciement de ta part, je suis encore à l'attendre ! (Remontant vers lui.) N'empêche que quand je suis rentrée, en transpiration, j'ai éprouvé le besoin de me mettre à l'aise. Je crois que c'est permis?

VENTROUX. - Eh bien ! oui, ça... ! ça, j'admets !

CLARISSE, remontant au-dessus du canapé. - C'est encore heureux ! Parbleu, tu es au frais, ici! Tu ne te doutes pas que dehors nous avons au moins... trente-cinq ou trente-six degrés... de latitude!

VENTROUX, ironique. - De latitude ?

CLARISSE, à qui l'intention de son mari échappe. - Trente-six degrés, parfaitement !

G. FEYDEAU, Occupe-toi d'Amélie, 1908

En (8), comme la didascalie et la réponse de Clarisse le signalent, celle-ci n'a pas perçu l'ironie contenue dans la réplique précédente de son mari. C'est que Ventroux sait que le terme « latitude » ne caractérise pas la température. C'est une connaissance d'ordre encyclopédique partagée avec les lecteurs ou spectateurs de la pièce. Mais Clarisse ne dispose pas de cette connaissance, elle ne réalise donc pas que son mari pointe son erreur.

(9) LE GÉNÉRAL, se retournant. - Non, comment ! te voilà toi ?... Bartholo a quitté Desdémone?

PETYPON. - Comme vous voyez!... (A part, avec ironie.) Bartholo avec Desdémone ! (Haut.) Hein ! Si Don Juan savait ça !...

LE GÉNÉRAL, gouailleur. - Ah! ah! «Don Juan et Desdémone !» tu es fort en littérature, toi !

PETYPON, s'inclinant ironiquement. - Vous me l'apprendrez.

LE GÉNÉRAL. - Je pourrais !... En attendant, tiens, puisque tu n'as rien à faire, montre donc la salle des Pastels à notre sous-préfet.

G. FEYDEAU, La Dame de chez Maxim, 1899

C'est le même cas de figure en (9), le général croit savoir, à tort, que Bartholo et Desdémone sont des personnages qui appartiennent à une même pièce de théâtre dans laquelle ils forment un couple. Il se méprend et cette erreur est repérée à la fois par Petypon et par les spectateurs/lecteurs de la pièce. Petypon par l'écho qu'il fait au discours du général pointe cette lacune littéraire pour les spectateurs dans un premier temps comme l'indique la didascalie «à part». Puis il poursuit le jeu ironique en mentionnant le personnage de Don Juan aux dépens du général qui n'est pas en mesure de percevoir l'ironie, ignorant qu'il est dans l'erreur. Si le général sait que Don Juan et Desdémone n'appartiennent pas à la même pièce de théâtre, il ne sait pas que Petypon produit volontairement cette confusion pour pointer d'autant plus la sienne. En se moquant de la lacune qu'il croit déceler chez son neveu par l'énoncé «tu es fort en 
littérature toi », le général se présente implicitement comme quelqu'un doté de connaissances littéraires en mesure de juger de la défaillance de son interlocuteur. L'ironie de la réplique « vous me l'apprendrez » de Petypon par laquelle il feint de ne pas être doté de culture littéraire ne peut qu'échapper au général.

Ainsi en (8), (9) la disparité des connaissances encyclopédiques des interlocuteurs, en (6) et (7), l'absence de connaissances contextuelles partagées, l'exclusion du cercle de connivence, font obstacle à la compréhension de l'ironie. Le socle commun de connaissances, ou « common ground » mis en avant par Clark et Gerrig (1984) est un élément fondamental sur lequel s'appuie la compréhension de l'ironie.

Il est des cas où l'interlocuteur fait le choix d'ignorer le sens implicite des propos tenus par l'ironisant.

\subsection{Non prise en compte volontaire de l'attaque contenue dans l'énoncé de L2.}

(10) JOE. - vous ne m'avez pas répondu. Selon vous, Isabelle...?

WANDA, l'interrompant. - franchement, Joë, voulez-vous que je vous donne un conseil ?

JOË, ironique. - un conseil d'amie, sans doute?

WANDA. - parfaitement.

JOË. - dites toujours

WANDA, âprement. - eh bien, vous feriez mieux de renoncer à tout ça

R. MARTIN DU GARD, Un Taciturne, 1932

Dans cette occurrence, la question de Joe « un conseil d'amie, sans doute ? » prend le sens de « ce ne peut pas être un conseil d'amie donc ce n'est pas un conseil au sens altruiste du terme ", et suggère aussi " ce que vous allez dire n'est certainement pas pour mon bien, étant donné que vous êtes loin d'être mon amie », ce qui constitue une critique de Wanda. Leur relation de conflits et de jalousie, voire de haine de la part de Wanda, est très bien installée, les deux personnages connaissent parfaitement les intentions de chacun envers l'autre. Elle ne sera jamais l'amie de Joe, pas plus qu'il ne pourrait être le sien. Wanda choisit donc d'ignorer le sens implicite de l'interrogation de Joe en produisant l'énoncé « parfaitement », qui est un adverbe à valeur de forte approbation. L'enchaînement de Joe «dites toujours », couplant le verbe dire à la deuxième personne du pluriel de l'impératif et l'adverbe toujours postposé, exprime l'idée que ce qui vient d'être dit par Wanda n'aura, en tout état de cause, aucune sorte d'effet; et marque son indifférence. Ainsi se dégage l'idée que Joe considère que ce qu'il va entendre aura peu d'influence sur lui, même si cette dernière continue à faire comme si elle voulait son bien. Wanda poursuit dans le ton prescripteur comme le suggèrent la didascalie «âprement» et l'emploi de la locution verbale "faire mieux de + Infinitif » à la deuxième personne du conditionnel par le biais de laquelle elle intime à Joe ce qu'il devrait faire.

\section{Réponse ambiguë}

L1, dans sa réponse, peut ne pas donner d'informations sur son interprétation de l'énoncé de L2. Dans la première configuration que nous observons, tout se passe comme si L2 n'était pas intervenu.

\subsection{L'enchaînement ne se fait ni sur la valeur directe ni sur la valeur indirecte de l'énoncé de L2}

(11) THIERRY. - ma chère Wanda, puisque l'occasion s'en présente...

WANDA, ironique. - ... par hasard...

THIERRY. - ... j'aime mieux vous avertir charitablement que vous faites fausse

route... que vous perdez votre peine en voulant jouer ce jeu-là.

R. MARTIN DU GARD, Un Taciturne, 1932

Dans l'exemple qui précède, il est difficile de savoir si Thierry a saisi la tonalité ironique avec laquelle Wanda produit l'énoncé «par hasard». Peut-être est-ce parce qu'elle se contente, explicitement du moins, de prolonger l'énoncé de Thierry «l'occasion s'en présente ». Ce type d'énoncé qui prend la 
forme d'une co-construction n'appelle pas forcément de tour suivant. La réplique suivante de Thierry «j'aime mieux... » constitue la proposition principale dont dépend la proposition subordonnée de son tour précédent «puisque l'occasion... ». Thierry achève donc son énoncé en lui donnant la complétude syntaxique qui lui manquait. Il n'enchaîne pas sur le sens implicite de la réplique de Wanda (« l'occasion ne s'en présente pas par hasard puisque c'est vous qui l'avez provoquée »). Wanda semble simplement avoir glissé la locution nominale «par hasard» sans que celle-ci n'ait perturbé, outre la pause temporelle, le discours de Thierry. L'intervention de Wanda n'est pas considérée comme venant modifier ce qu'il a à dire. Elle précise uniquement une circonstance de l'énonciation et la commente.

Il est des cas où l'enchaînement sur l'énoncé ironique laisse planer un autre type d'ambiguïté : la question demeure de savoir si l'interlocuteur enchaîne sur la valeur directe de l'énoncé ou sur sa valeur indirecte d'attaque. C'est ce qui se produit dans l'occurrence qui suit :

\subsection{Réplique de L1 interprétable à la fois comme enchaînement sur la valeur directe et sur la valeur indirecte de l'énoncé de L2}

Cette ambiguïté peut être due à la combinaison d'indices d'orientations contradictoires ou bien à l'absence d'indices orientés.

\subsection{1 - Ambiguïté due à la combinaison d'indices d'orientations contradictoires}

(12) TRIELLE. - [...]attendant qu'elles te devinssent agréables ; je dus passer à un autre genre d'exercice. C'est alors que j'imaginai de me venger sur le mobilier.

VALENTINE, ironique. - C'était malin.

TRIELLE. - Très malin même, puisque le jour où d'un coup de tabouret je fis voler en éclats le miroir de l'armoire à glace, tu restas muette d'ahurissement, de quoi j'éprouvai une joie telle qu'en moins de six semaines j'immolai sans regret, à mon ardente soif de silence, deux chaises, le pot à eau, le casier à musique, la lampe, la pendule, la soupière, le buste de ton oncle Arsène (orgueil de notre humble salon), et divers autres objets de première nécessité. Le fâcheux est, ô Valentine, qu'il n'en soit pas du mobilier comme du phénix qui renaît de ses cendres. La perspective d'avoir à en acheter d'autre me gâta vite l'âpre jouissance que je goûtais à casser les meubles ; une fois encore je dus chercher autre chose. Seulement quoi ? M'en aller ? Peut-être. Mais où aller? Car tout est là pour un homme dont les goûts bourgeois répugnent au, concubinage comme à la triste vie d'hôtel. [...]

VALENTINE. - Quand tu auras assez causé, tu le diras.

G. COURTELINE, La Paix chez soi, 1906

Valentine en répondant à son mari «c'était malin » ${ }^{i i}$ se moque de l'énoncé que ce dernier vient de prononcer «c'est alors que j'imaginai de me venger sur le mobilier ». Le mari cherche une façon de faire payer à sa femme l'attitude désagréable qu'elle manifeste envers lui. Après s'être essayé à lui donner la fessée, il utilise une autre stratégie : « se venger sur le mobilier ». Ce choix est perçu comme absurde aux yeux de Valentine : Trielle a déboursé en effet beaucoup d'argent pour remplacer le mobilier qu'il a luimême détérioré. Il a donc fait, selon elle et selon la logique, un mauvais calcul car il est au final celui qui a payé les "pots cassés », c'est le cas de le dire. Quand elle utilise l'adjectif subjectif axiologique positif malin, c'est pour faire écho à la façon qu'a Trielle de présenter son raisonnement.

Il présente en effet sa vengeance sur le mobilier comme le fruit d'un véritable processus intellectuel « c'est alors que j'imaginai », comme le suggèrent le verbe de pensée " imaginer » et l'adverbe alors qui marque l'aboutissement de ce processus réflexif. Il donne ainsi à son acte l'allure d'une stratégie, d'une opération « habile » et réfléchie en vue de parvenir à un but. Or « se venger sur le mobilier » est une action qui a eu des conséquences fâcheuses pour lui, qu'il aurait pu mesurer et auxquelles il semble n'avoir pas songé. L'énoncé de Valentine prend ainsi implicitement le sens de l'assertion négative correspondante «ce n'était pas malin » et constitue une critique de son mari et de son raisonnement. Trielle saisissant ce sens implicite et l'attaque contenue dans les propos de sa femme choisit de rebondir sur l'adjectif malin «c'était très malin même », en renchérissant par l'adverbe d'intensité très ainsi que 
par l'adverbe même. Son énoncé se présente ainsi comme une rectification de l'énoncé de sa femme et coorienté à son sens explicite. Cependant, il ne se contente pas de produire un énoncé co-orienté et renchérissant celui de sa femme, il justifie ce renchérissement par une subordonnée de cause introduite par la conjonction de subordination puisque. Cette justification suggère que Trielle estime que sa femme n'était pas convaincue que son plan était effectivement malin. Il y a en effet argumentation, quand il y a désaccord (Moeschler, 1980). C'est ce qui nous invite à penser que Trielle enchaîne en réalité sur le sens implicite négatif ( « ce n'était pas malin ») de l'énoncé de Valentine. La suite de son énoncé décrit le plaisir qu'il a éprouvé à se venger de la sorte «tu restas muette d'ahurissement, de quoi j'éprouvai une joie telle qu'en moins de six semaines j'immolai sans regret... ». Il démontre ainsi à sa femme que son plan a bien atteint son objectif. L'adjectif malin dans son discours est à prendre dans son sens plein, premier de "rusé ", " astucieux ». Il nous semble surtout que Trielle prend Valentine au piège de ses mots : celle-ci en utilisant l'ironie offre, malgré elle, l'opportunité à Trielle de recevoir son énoncé comme une appréciation positive et d'ignorer sa critique, non inscrite explicitement. Trielle joue avec l'énoncé de sa femme, en redonnant son sens plein à l'adjectif malin.

On a vu dans cet exemple un type d'enchaînement ambivalent : la réponse formulée par Trielle après l'énoncé ironique est ambiguë, par certains aspects elle semble être un enchaînement sur la valeur explicite de l'énoncé de Valentine (Trielle utilise l'adjectif malin dans son sens premier de rusé et renchérit avec l'adverbe même) et à la fois un enchaînement sur sa valeur indirecte (Trielle se justifie comme s'il n'était pas évident pour Valentine que son idée de se venger sur les meubles ait été astucieuse).

C'est exactement le même type de configuration en (13) :

(13) LE PRÉSIDENT. - Vous n'avez jamais eu de condamnations ?

LA BRIGE. - jamais.

LE PRÉSIDENT. - ça m'étonne.

LA BRIGE. - je vous crois sans peine ; mais je suis un gaillard habile.

LE PRÉSIDENT, ironique. - soit dit sans vous flatter.

LA BRIGE. - sans me flatter, en effet, puisque j'ai résolu le difficile problème de pouvoir, à trente-six ans, justifier à la fois et d'un passé sans tâche, et d'un casier judiciaire sans souillure.

LE PRÉSIDENT. - voilà de bien grands mots : mettons les choses au point. Vous n'avez jamais eu de condamnations, c'est vrai, mais les renseignements recueillis sur votre compte ne sont guère en votre faveur. Ils vous représentent comme un personnage de commerce presque impossible, comme une façon de Chicaneau, processif, astucieux, retors, éternellement en bisbille avec le compte courant de la vie. Les juges ne sont occupés qu'à trancher vos petits différends avec le commun des mortels, et les archives des commissariats regorgent de procès-verbaux dont votre nom fait les frais

G. COURTELINE, L'Article 330, 1900

La Brige vient de se décrire lui-même comme «un gaillard habile », syntagme nominal contenant l'adjectif axiologique positif « habile », ce qui constitue un auto-ffa, un acte de flatterie envers lui-même. Le président catégorise immédiatement cet auto-compliment comme un acte de parole non flatteur. La Brige enchaîne en confirmant les paroles du président par la locution adverbiale «en effet » et en justifiant qu'il ne s'agit effectivement pas d'un auto-compliment. Comme dans le cas précédent, le fait que La Brige éprouve le besoin d'argumenter ce qu'il vient d'affirmer et ce que vient d'exprimer explicitement le président montre qu'il ne va pas de soi qu'il ne vient pas de se flatter. L'affirmation du président si elle était à prendre dans son sens plein n'aurait pas besoin d'être justifiée par La Brige, on ne peut, en effet, convaincre que quelqu'un qui n'est pas déjà persuadé du bien-fondé de son affirmation.

Dans les deux exemples précédents l'enchaînement contenait à la fois des indices co-orientés à la valeur directe de l'énoncé ironique ( « en effet », "même ») et des indices anti-orientés à cette valeur directe (la justification qui constitue une argumentation et présuppose un désaccord). Dans l'occurrence suivante, l'ambiguïté découle d'une absence de signes clairement orientés. 


\subsection{2 - Absence d'indices orientés dans l'enchaînement}

(14) IDA avec effort. - Alors voici ! Dans un cas aussi... préoccupant, je crois, enfin j'imagine... qu'il serait bon... de consulter l'homme qui vous a permis de transformer notre société d'une façon aussi extraordinaire!

LE PROMOTEUR. - Quoi ? Le professeur Buisson? L'inventeur du Sérum de l'Insomnie ? (Avec brusquerie) Il est bien où il est.

IDA, avec une ironie amère. - Oui, dans sa propre clinique, aux mains de ses propres infirmiers !

LE PROMOTEUR, chantonnant avec un affreux mauvais goût. - On n'est jamais si bien qu'au sein de sa famille !

J.TARDIEU, La Cité sans sommeil, 1984

L'enchaînement du Promoteur «On n'est jamais si bien qu'au sein de sa famille!» peut s'interpréter comme une opposition au sens sous-entendu de la réplique d'Ida « il n'est pas bien là où il est ». Il s'agirait d'une opposition qui ne s'exprime pas par un adverbe de négation frontale comme dans «(non détrompez-vous) on est jamais si bien qu'au sein de sa famille ».

Cet énoncé du promoteur peut tout aussi bien être compris comme renchérissant l'énoncé explicite d'Ida, « il est bien là où il est » et prendre le sens de [(parfaitement, surtout qu'[on n'est jamais si bien qu'au sein de sa famille]. L'énoncé du promoteur prolonge d'ailleurs du point de vue sémantique celui d'Ida. Cette dernière en utilisant l'adjectif "propres » et les déterminants possessifs « sa » et « ses » insiste sur le lien d'appartenance qui unit le professeur à la clinique et au personnel. Cette dimension d'appartenance, de proximité et de possession est reconduite dans le syntagme nominal «sa famille » contenant le déterminant possessif «sa ». On peut y entendre le substantif «famille» au sens large, de personnes avec qui on a tissé des liens forts, étroits. Le terme prend le sens des «siens ». Cependant, l'absence d'adverbe marquant explicitement l'accord ou le désaccord avec l'énoncé antérieur empêche une interprétation univoque de cet enchaînement.

Dans le cas suivant, l'enchaînement se fait sur la valeur directe de l'énoncé ironique, par inadvertance, puis sur sa valeur indirecte.

\subsection{Enchaînement successif sur la valeur directe puis indirecte de l'énoncé ironique}

Nous voyons avec le cas suivant le processus de « miroir » à l'œuvre dans l'ironie.

(15) MONSIEUR . - Comme c'est curieux ! Il me disait, à l'instant même, que ce travail-là lui rappelait le régime pénitentiaire.

MADAME. - Ah il te disait ça ?... l'ingrat !

MONSIEUR faussement naïf. - Ingrat, pourquoi donc ? Ce n'est pas toi qui lui fais ses paniers je suppose?

MADAME pensant à autre chose. - Bien sûr que si! (se reprenant) Qu'est-ce que je dis ? Non, bien sûr, ce n'est pas moi qui lui fais ses paniers, mais c'est moi, enfin c'est nous qui...

MONSIEUR, l'interrompant avec ironie. - C'est nous qui lui fournissons l'osier du jardin, n'est-ce pas? Mais alors, dis donc, nous devrions avoir une part sur les bénéfices?

MADAME, étourdiment. - Bien sur !

MONSIEUR sursautant. - Comment, bien sur?

MADAME paraissant assez embarrassée. - Enfin je veux dire...(regardant vers la gauche) taisons-nous, le voici !

J. TARDIEU, Le Petit voleur rêve de banlieue, 1984

L'ironie reprend parfois la forme linguistique de l'autre, l'écho peut être dans ce cas littéral mais il peut aussi ne faire que représenter sous forme d'énoncé la logique, l'opinion de l'autre sans que l'interlocuteur n'ait prononcé explicitement cet énoncé. Cet exemple relève de ce dernier type. Monsieur ne reprend pas 
un énoncé littéralement présent dans le discours de Madame mais représente verbalement la logique qu'il lui prête, la conclusion qu'elle aurait pu formuler selon son propre schéma déductif.

L'enchaînement de Madame «bien sûr » nous indique qu'elle a d'abord répondu à l'énoncé de Monsieur «mais alors, dis donc nous devrions avoir une part sur les bénéfices? " comme s'il n'avait pas de sens implicite. Cet énoncé de Monsieur correspond à la logique de Madame, elle répond donc par l'énoncé de confirmation «bien sûr!", dont l'exclamation suggère son engouement à acquiescer à un énoncé qui va de soi selon son propre raisonnement. Monsieur renvoie en miroir les conclusions que Madame aurait tirées elle-même. Mais cette réponse de Madame l'étonne comme l'indiquent la didascalie « sursautant », la question contenant l'adverbe interrogatif «comment» et la reprise de la confirmation de Madame «bien sûr ». Monsieur ne s'attendait pas à ce type de réponse. Son énoncé était destiné à critiquer le raisonnement de sa femme, pas à le conforter. Madame pensant que son mari partageait son jugement, aveuglée par son désir de richesse, a été troublée et n'a pas entendu le sens implicite de sa question. La didascalie «étourdiment » suggère en effet un moment d'égarement pendant lequel elle a perdu quelque peu conscience. Le début de la scène présente d'ailleurs Madame comme distraite et revenant sur ses paroles par des auto-rectifications («bien sûr que si! (se reprenant) Qu'est-ce que je dis ? Non bien sûr »). Elle saisit après coup son erreur, erreur qui se lit à la fois à travers la didascalie « paraissant assez embarrassée » et la boucle méta énonciative «enfin je veux dire » qui initie sa réplique suivante. Elle s'apprêtait à produire un énoncé rectificatif par lequel elle serait revenue sur son dire. Monsieur a ainsi trop bien imité, reproduit la logique de sa femme dans son énoncé et alors qu'il n'y adhérait pas, cette non-adhésion n'a pas été reconnue par elle et a mené cette dernière vers un aveu involontaire. Charmée, bercée par le discours-miroir que lui tenait son mari, se sentant enfin comprise et suivie dans sa logique, elle n'y a pas perçu l'ironie puis a fini par revenir à la réalité du sens de l'énoncé produit.

\subsection{Ambiguïté de l'enchaînement due au prolongement par L1 du jeu ironique}

Enfin, l'enchaînement peut être ambigu car l'interlocuteur poursuit le jeu ironique initié par son partenaire d'interaction.

(16) FRONTIN . - Et que savez-vous si je voudrai de lui ?

LISETTE. - Madame a raison, tu ne lui ferais pas là un grand présent.

ARAMINTE. - Vous parlez fort mal, Lisette ; ce que j'ai répondu à Frontin ne signifie rien contre Ergaste, que je regarde comme un des hommes les plus dignes de l'attachement d'une femme raisonnable.

LISETTE, d'un ton ironique. - À la bonne heure; je le trouvais un homme fort ordinaire, et je vais le regarder comme un homme fort rare.

FRONTIN. - Pour le moins aussi rare que ta maîtresse (soit dit sans préjudice de la reconnaissance que j'ai pour la bonne chère que j'ai faite chez elle).

DORANTE. - Halte-là, faquin ; prenez garde à ce que vous direz de Madame la

Marquise.

FRONTIN. - Monsieur, je défends mon maître.

MARIVAUX, Les Sincères, 1739

Dans cette occurrence, Lisette affirme que son point de vue sur Ergaste, maître de Frontin, a changé du tout au tout. Elle utilise l'adjectif « rare » explicitement dans un sens axiologique positif. Implicitement, elle fait entendre que son point de vue n'a pas changé juste parce qu'Araminte vient de louer Ergaste : son jugement antérieur reste d'actualité, elle estime qu'il est un homme « fort ordinaire ». L'adjectif « rare » dans sa bouche prend ainsi le sens opposé de banal, commun. Frontin réutilise l'adjectif axiologique explicitement positif « rare» pour qualifier cette fois la maîtresse de Lisette, dans une comparaison d'égalité « aussi rare que ta maîtresse ». Explicitement il semble donc enchaîner sur la valeur positive du dit de Lisette mais il ne fait que prolonger le jeu ironique de cette dernière. Dans son discours, l'adjectif rare revêt également une valeur négative. Ceci est indiqué d'une part, par la suite de l'énoncé qui contient une boucle méta-énonciative "soit dit sans préjudice » venant prévenir qu'il ne faut pas voir dans son dire de l'ingratitude vis-à-vis de la marquise qui l'a bien reçu et d'autre part par l'enchaînement de Dorante : « halte-là, faquin; prenez garde à ce que vous direz de Madame la Marquise » qui contient l'injure « faquin », terme axiologique négatif adressé à Frontin, et un acte d'avertissement sur les propos 
que ce dernier tient sur la marquise. Cet acte suppose que Dorante a interprété les propos précédents de Frontin comme une attaque de la marquise. Enfin Frontin catégorise lui-même ses propres propos comme une défense de son maître «je défends mon maître » qui nous indique qu'il attaque la marquise pour répondre à ce qu'il avait reçu comme une attaque initiale de la part de Lisette. Ainsi Frontin a bien perçu la valeur négative implicite du dit de Lisette et a bien enchaîné sur celle-ci par une attaque en retour. Mais cette attaque a emprunté les mêmes couleurs que celle de Lisette : à une comparaison explicitement positive utilisant l'adjectif axiologique mélioratif « rare »s'attachait une valeur négative implicite. Le jeu ironique s'est déployé à travers le même lexème et différents locuteurs, cependant dans la réplique de Frontin l'ironie n'est pas signalée de façon métadiscursive dans les didascalies.

\section{Conclusion}

Le corpus théâtral permet une analyse fine des phénomènes interlocutifs. On peut ainsi y percevoir l'énoncé catégorisé comme ironique au sein d'un échange de trois tours: l'énoncé ironique apparaît toujours comme une intervention réactive de l'ironisant, produite suite à une intervention initiative de l'ironisé. L'intervention initiative de L1 est interprétée par l'ironisant comme manquant de pertinence. L'ironisant, L2, veut alors pointer ce manque de pertinence. Il s'agit de mettre l'autre en face de ses contradictions. L'énoncé ironique acquiert de fait toujours une valeur d'attaque qui n'est jamais directe. Dans le cadre de cet article, nous nous sommes concentrée sur le troisième tour de parole, celui suivant la réplique ironique.

Nous avons observé que divers enchaînements peuvent être produits suite à cet énoncé se voulant ironique. Ce pointage des contradictions peut ainsi ne pas du tout être perçu par l'ironisé, qui se contente d'enchaîner sur la valeur directe de l'énoncé. Il peut choisir d'ignorer la valeur indirecte d'attaque. Il peut enfin enchaîner sur cette valeur dérivée et même prolonger le jeu ironique de sorte à renvoyer l'attaque à l'ironisant. Enfin, pour être reconnue, l'attaque suppose un socle commun de connaissances appartenant au non-dit qu'elle réactive implicitement: si celui-ci n'est pas partagé par les participants, l'attaque ne peut pas aboutir.

Tout repose dans l'énonciation ironique sur le non-dit qui entraîne forcément des non-compréhensions, des ambiguïtés, des jeux subtils et rebondissements sur le sens des mots prenant parfois au piège celui qui se croyait préservé par l'indirection.

\section{Bibliographie}

Barthes, R., Berthet, F. (1979). Présentation. Communications, 30, 3-5.

Berrendonner, A. (1981). De l'ironie, Eléments de pragmatique linguistique. Paris : Minuit, 173-239.

Bres, J. (2010). L'ironie, un cocktail dialogique? Actes du 2ème Congrès Mondial de Linguistique

Française. DOI: $10.1051 / \mathrm{cmlf} / 2010093$.

Clark, H., Gerrig, R. (1984). On the pretense theory of irony. Journal of Experimental Psychology General, 113, $121-126$.

Currie, G. (2006). Why irony is pretence. Ed Shaun. N, The Architecture of the imagination. Oxford: Oxford university press, 111-135.

Ducrot, O. (1984). Esquisse d'une théorie polyphonique de l'énonciation. Le dire et le dit. Paris : Minuit, 171-233.

Dumarsais. (1730/ 1988). Des Tropes. Paris : Flammarion.

Fontanier, P. (1830 / 1977). Les Figures du discours. Paris : Flammarion.

Kerbrat-Orecchioni, C. (1980). L'ironie comme trope. Poétique, 41, 108-127.

Kerbrat-Orecchioni, C. (1986). L'Implicite. Paris : Armand Colin. 
Martin, R. (1983). Pour une logique du sens. Paris : Presses universitaires de France.

Moeschler, J. (1980). La réfutation parmi les fonctions interactives marquant l'accord et le désaccord. Cahiers de Linguistique Française, 1, 54-78

Perrin, L. 1996. L'Ironie mise en tropes : du sens des énoncés hyperboliques et ironiques. Paris : Kimé.

Rabatel, A. (2012). « Ironie et sur-énonciation ». Vox romanica, 71, 42-76.

Quintilien, M. (95/ 1978). De Institutione oratoria. Paris : Belles Lettres.

Sperber, D. et Wilson, D. (1978). Les ironies comme mention. Poétique, 36, 399-412.

i Trésor de la langue française informatisé : http://atilf.atilf.fr/

${ }^{\text {ii }}$ On peut s'interroger sur la lexicalisation de ce tour dans la mesure où on trouve au sein de la définition concernant l'adjectif malin dans le dictionnaire (TLFi) une entrée pour son sens antiphrastique qui équivaudrait à : «ce n'est pas très intelligent ». 